\title{
Foveal Structure and Visual Function in Nanophthalmos and Posterior Microphthalmos
}

Philipp L. Müller ${ }^{1,2,3}$, Tim Treis ${ }^{4}$, Abdulrahman Alsaedi ${ }^{1,5}$, Andrew R. Webster ${ }^{1,6}$, Peng Khaw $^{1,6}$, Michel Michaelides ${ }^{1,6}$, Louisa Wickham ${ }^{1,6}$, Dilani Siriwardena ${ }^{1}$, Paul Foster ${ }^{1,6}$, Mariya Moosajee ${ }^{1,6,7,8}$, Carlos Pavesio ${ }^{1}$, Adnan Tufail ${ }^{1,6}$, Catherine Egan ${ }^{1,6,{ }^{*}}$

${ }^{1}$ Moorfields Eye Hospital NHS Foundation Trust, London, UK

${ }^{2}$ Department of Ophthalmology, University of Bonn, Bonn, Germany

${ }^{3}$ Center for Rare Diseases, University of Bonn, Bonn, Germany

${ }^{4}$ BioQuant, University of Heidelberg, Heidelberg, Germany

${ }^{5}$ College of Medicine, Imam Mohammad Ibn Saud Islamic University, Riyadh, Saudi

Arabia

${ }^{6}$ Institute of Ophthalmology, University College London, London, UK

${ }^{7}$ Great Ormond Street Hospital for Children, London, UK

${ }^{8}$ The Francis Crick Institute, London, UK

Word Count: 2971

Figures: Figures 1, 2, 3, 4

Tables: Table 1

Online Supplementary Material:

Supplementary Results; Supplementary Figures S1, S2, S3; Supplementary Tables S1, S2, S3, S4

\section{Keywords:}

Retina; Fovea; Microphthalmos; Nanophthalmos; Optical coherence tomography; Imaging; Visual Acuity

\author{
${ }^{*}$ Corresponding author: \\ Catherine Egan \\ Moorfields Eye Hospital NHS Foundation Trust, \\ 162 City Rd \\ London EC1V 2PD \\ United Kingdom \\ Telephone: +44207566 2576 \\ E-mail: cathy.egan@ucl.ac.uk
}




\section{SYNOPSIS/PRECIS}

This study reveals that demographic, clinical and imaging parameters have significant impact on visual acuity in nanophthalmos or posterior microphthalmos. It might give further insights into the pathophysiology, foveal development and emmetropisation. 


\section{ABSTRACT}

Background/Aims: The reason for visual impairment in patients with nanophthalmos and posterior microphthalmos is not completely understood. Therefore, this study aims to investigate foveal structure, and the impact of demographic, clinical, and imaging parameters on best-corrected visual acuity (BCVA) in these conditions.

Methods: Sixty-two eyes of 33 patients with nanophthalmos $(n=40)$ or posterior microphthalmos $(n=22)$, and 114 eyes of healthy controls with high-resolution retinal imaging including spectral-domain or swept-source optical coherence tomography (OCT) images were included in this cross-sectional case-control study. Foveal retinal layer thickness was determined by two independent readers. A mixed effect model was used to perform structure-function-correlations and predict the BCVA based on subjectspecific variables.

Results: Most patients (28/33) had altered foveal structure associated with loss of foveal avascular zone and impaired BCVA. However, widening of outer nuclear layer, lengthening of photoreceptor outer segments, normal distribution of macular pigment, and presence of Henle fibres were consistently found. Apart from the presence of choroidal effusion, which had significant impact on BCVA, the features age, refractive error, axial length, and retinal layer thickness at the foveal centre explained $61.7 \%$ of the variability of BCVA.

Conclusion: This study demonstrates that choroidal effusion, age, refractive error, axial length, and retinal layer thickness are responsible for the majority of interindividual variability of BCVA as well as the morphologic foveal heterogeneity in patients with nanophthalmos or posterior microphthalmos. This might give further insights into the physiology of foveal development, the process of emmetropisation and support clinicians in the assessment of these disease entities. 


\section{INTRODUCTION}

Nanophthalmos and posterior microphthalmos are rare, bilateral developmental eye disorders, which present either as an isolated abnormality or as part of a syndrome, and either sporadically or in an inherited manner (autosomal-dominant or autosomalrecessive).[1-11] Both disorders are defined by compromised growth of the posterior segment, leading to short axial length (usually $\leq 20 \mathrm{~mm}$ ) associated with high hyperopia (usually $\geq 8.00$ dioptres).[3,12] In contrast to posterior microphthalmos, patients with nanophthalmos also present a shallow anterior chamber.[12] By definition, there are no other obvious structural defects. However, thickened sclera and a high lens/globe volume ratio, as well as complications including narrow angle glaucoma and choroidal effusion have been described.[13]

The best-corrected visual acuity (BCVA) in patients affected by these disease entities is often worse than 0.2 LogMAR (20/32 Snellen equivalent).[14] The reason for this visual impairment is not completely understood. Foveal hypoplasia, "macular folds", and a rudimentary foveal avascular zone (FAZ) have been described in some cases of nanophthalmos or posterior microphthalmos, using optical coherence tomography (OCT) and/or fluorescein angiography.[3,14-19] However, a comprehensive and indepth evaluation of foveal structure in a larger cohort of patients with nanophthalmos or posterior microphthalmos using current imaging technology is still required.

Therefore, we systematically examined patients with nanophthalmos and posterior microphthalmos using an up-to-date multimodal imaging approach. The aim of this study was to investigate and describe (i) morphologic ocular alterations including retinal microstructure, (ii) associations with diagnosis and/or disease severity, and (iii) structure-function-correlation. This might help to improve knowledge of underlying pathophysiology, as well as patient assessment and counselling. 


\section{MATERIALS AND METHODS}

This retrospective observational case series was performed at Moorfields Eye Hospital NHS Foundation Trust, London, United Kingdom. Patients with nanophthalmos and posterior microphthalmos were identified by text search of electronic medical records from Moorfields Eye Hospital between 2004 and 2019. The inclusion criteria were defined as (1) axial lengths $\leq 20 \mathrm{~mm}$ and/or hyperopia $\geq 8.00$ dioptres refractive error (spherical equivalent) associated with nanophthalmos or posterior microphthalmos (divided by the presence of shallow anterior chamber; threshold set at $2.5 \mathrm{~mm}$ depth), and (2) spectral domain or swept source OCT images adequate to evaluate the fovea (e.g., central alignment and sufficient image quality). Sixty-eight eyes of 34 patients met the inclusion criteria. In terms of disease-associated complications, we evaluated narrow angle glaucoma and choroidal effusion. Previous retinal surgery or other pathologies known to affect visual acuity and/or OCT measurements were excluded. In our cohort, this was the case in six eyes of five patients with concomitant pathologies as follows: uveitis in two cases, amblyopia in one case, neovascular age-related maculopathy in one case, and status post retinal detachment in both eyes of one patient. A normative database of the Department of Ophthalmology, University of Bonn, Germany served as controls. Institutional review board approval (Ethikkommission, Medizinischen Fakultät, Rheinische Friedrich-Wilhelms-Universität Bonn, Germany; approval ID, 027/07) and subject's written informed consent were obtained. The study was in adherence with the declaration of Helsinki.

\section{Genetic testing}

Genetic data was available in seven patients. Four probands (GC7258, GC27868, GC30420, and GC29302) underwent Sanger sequencing of all coding exons and intronexon boundaries of the Membrane-Type Frizzled-Related Protein gene (MFRP, Online Mendelian Inheritance in Man \#606227) as part of a research project (MOOA1016 12/LO/0141, Genetic study of inherited retinal eye disease, principal investigator ARW) and aspects of their condition have previously been reported.[17,20] One proband (GC 38302) underwent next-generation sequencing of a panel of genes as part of clinical testing (Manchester Centre for Genomic Medicine, Manchester, UK). Two probands (25866 and GC35426) were part of the 100000 Genomes project and underwent whole genome sequencing.[21] A further proband has undergone clinical genetic testing (North Thames Genomics Laboratory Hub, London, UK) and results are awaited.

\section{Imaging}

All participants had undergone an ophthalmic examination as part of their routine eye clinic visits. Prior to multimodal imaging, pupils were dilated. OCT was performed in all participants using an spectral-domain OCT (Spectralis HRA+OCT, Heidelberg Engineering, Heidelberg, Germany). A swept source device (AngioPlex, Carl-ZeissMeditec, Inc., Dublin, CA, USA) was used for OCT-Angiography (OCT-A). Scleral thickness was measured based on horizontal $10 \mathrm{MHz}$ ultrasound $\mathrm{B}$-scans covering the posterior pole. When available, infrared-reflectance and short-wavelength fundus autofluorescence (AF) imaging (488 $\mathrm{nm}$ excitation and 500-700 $\mathrm{nm}$ emission) was 
performed using a confocal scanning laser ophthalmoscope (cSLO; Spectralis $\mathrm{HRA}+\mathrm{OCT}$ ). In cases with measurement of macular pigment optical density (MPOD), it was determined with the two-wavelength AF method using a modified HRA classic (Heidelberg Engineering) as described elsewhere.[22-25] Axial length and anterior chamber depth was measured using the IOL-Master 500 (Carl Zeiss Meditec, Jena, Germany) or a-scan ultrasound.

\section{Image Processing}

To minimize the influence of cataract on visual acuities and the statistical evaluation, patient records were reviewed for description of lens status and unexplained changes in refraction and BCVA at any time point, and images were reviewed for shadowing suggestive for lens opacification. No eye was excluded based on this approach. Imaging data was then evaluated and correlated to demographic, functional and morphologic features. Central retinal structure was classified into three types based on the foveal depression in the central OCT scan (Figure 1): Regular foveal structure featuring extrusion of the inner retinal layers (IR), foveal pit, widening of the outer nuclear layer $(\mathrm{ONL})$, and lengthening of the photoreceptor outer segments was assigned to type 1 . Widening of the ONL, and lengthening of the photoreceptor outer segments associated to incomplete and absence of extrusion of IR were represented by type 2 and 3 , respectively. Thickness of full retina, IR, outer retina (OR), ONL, and photoreceptor inner and outer segments as well as RPE cells (ISOSRPE) were manually measured in OCT scans at the location of the presumed fovea (i.e., location of ONL widening and lengthening of the photoreceptor outer segments in the central macula) using the respective device inbuilt measurement tool (Figure 1). All measurements and classifications have been performed by two independent experienced readers masked to the results of each other and the patients' diagnosis. For further analysis, the mean of both readers was used.

\section{Statistical analysis}

Statistical analysis was performed using the software environment $R$ (version 3.2.3, The R Foundation for Statistical Computing, Vienna, Austria) and Python (version 3.7, Python Software Foundation. Python Language Reference).[26] Since the number of samples did not justify the assumption of the central limit theorem and many subpopulations were not sufficiently normal in Shapiro-Wilk tests, non-parametric tests were used. For comparison of two distributions, the Mann-Whitney $U$ test was used and for comparing more than two, the Kruskal-Wallis $\mathrm{H}$ test was used. P-values with $\alpha<$ 0.05 were considered statistically significant.

To investigate the influence of the individual features on the visual acuity, a linear regression analysis was performed. Since the data contained one observation per patient, an ordinary least squares (OLS) model was chosen for further analysis over a mixed effect model. If both eyes have been assessed for one patient, these were averages so that all data points are fully independent from each other. For the model, the measurements were scaled to unit variance to compensate for values coming from different domains (like age in years or thickness measures in $\mu \mathrm{m}$ ). Then, all possible 
combinations of the features were generated, adding a constant to center the error term of the OLS model. For each combination, a separate OLS model was fit using a k-fold cross-validation with $\mathrm{k}=10$. The models were ranked by their average mean squared error (MSE) in predicting the test data set across all ten folds in order to obtain the optimal model. Then, the residuals of the OLS model were investigated for systematic tendencies in order to assess the quality of the model. A possible serial correlation was investigated by randomly shuffling the data, but could not be found.

For inter-reader correlation, intraclass correlation coefficients (ICC, two-way random, absolute agreement), 95\% coefficients of repeatability (CR) and coefficients of variation (CV) were determined.[27,28] Bland-Altman plots were generated for visualization of limits of agreement. Spearman's rank correlation coefficients $(\rho)$ were calculated between the absolute differences and the mean values to determine whether measurement variability increases with lesion size.[28] 


\section{RESULTS}

\section{Cohort characteristics}

A total of 62 eyes of 33 patients (22 female, 11 male) with a mean ( \pm standard deviation, SD) age of $36.5 \pm 18.7$ years (range, 7.4-75.4 years) and 114 eyes of healthy controls (73 female, 41 male) with a mean age of 39.8 \pm 15.6 years (range, 8.0-64.6 years) were included. Forty eyes of 22 patients ( 17 female, 5 male) with a mean age of 41.8 \pm 17.4 years (range, 12.9-75.4 years) were considered to have nanophthalmos, and 22 eyes of 11 patients ( 5 female, 6 male) with a mean age of $25.7 \pm 16.6$ years (range, 7.4-58.4 years) were diagnosed with posterior microphthalmos (Table 1 and Supplementary Table S1). If both eyes of a patient were included (in 29 of 33 patients), they were assigned to the same disease entity. Comprehensive genetic data can be found in Supplementary Results and Supplementary Table S2.

\section{Table1: Demographic, clinical, and imaging parameters of included subjects}

\begin{tabular}{|c|c|c|c|c|c|c|c|c|}
\hline & & Controls & All patients & Nanophthalmos & $\begin{array}{c}\text { Posterior } \\
\text { Microphthalmos }\end{array}$ & $\begin{array}{c}\text { Type } 1 \\
\text { Foveal } \\
\text { Structure }\end{array}$ & $\begin{array}{c}\text { Type } 2 \\
\text { Foveal } \\
\text { Structure }\end{array}$ & $\begin{array}{c}\text { Type } 3 \\
\text { Foveal } \\
\text { Structure }\end{array}$ \\
\hline \multirow{3}{*}{$\begin{array}{l}\text { Patient } \\
\text { based }\end{array}$} & Number (n) & 114 & 33 & 22 & 11 & 5 & 13 & 15 \\
\hline & Sex (female/male) & $73 / 41$ & $22 / 11$ & $17 / 5$ & $5 / 6$ & $4 / 1$ & $9 / 4$ & $9 / 6$ \\
\hline & Age (years) ${ }^{a}$ & $39.8 \pm 15.6$ & $36.5 \pm 18.7$ & $41.8 \pm 17.4$ & $25.7 \pm 16.6$ & $41.0 \pm 23.8$ & $36.3 \pm 16.3$ & $35.1 \pm 18.5$ \\
\hline \multirow{14}{*}{$\begin{array}{l}\text { Eye } \\
\text { based }\end{array}$} & Number (n) & 114 & 62 & 40 & 22 & 9 & 26 & 27 \\
\hline & Diagnosis (N/PM, n) & $0 / 0$ & $40 / 22$ & $40 / 0$ & 0/22 & $7 / 2$ & $16 / 10$ & $17 / 10$ \\
\hline & BCVA (LogMAR) a & $0.0 \pm 0.0$ & $0.55 \pm 0.51$ & $0.50 \pm 0.37$ & $0.64 \pm 0.68$ & $0.11 \pm 0.11$ & $0.64 \pm 0.61$ & $0.61 \pm 0.39$ \\
\hline & Refractive error (dpt) $a, b$ & $-0.22 \pm 2.21$ & $15.85 \pm 3.76$ & $15.39 \pm 3.99$ & $16.67 \pm 3.12$ & $12.31 \pm 2.15$ & $14.62 \pm 3.30$ & $18.20 \pm 3.03$ \\
\hline & Axial length $(\mathrm{mm})^{\mathrm{a}}$ & $23.07 \pm 0.73$ & $16.52 \pm 1.29$ & $16.72 \pm 1.48$ & $16.15 \pm 0.69$ & $18.87 \pm 0.82$ & $16.34 \pm 0.92$ & $15.91 \pm 0.72$ \\
\hline & $\begin{array}{l}\text { Anterior chamber } \\
(\mathrm{mm})^{\mathrm{a}}\end{array}$ & $3.50 \pm 0.31$ & $2.17 \pm 0.58$ & $1.80 \pm 0.33$ & $2.86 \pm 0.17$ & $2.13 \pm 0.42$ & $2.20 \pm 0.55$ & $2.16 \pm 0.65$ \\
\hline & ОСТ type $(1 / 2 / 3 ; n)$ & $114 / 0 / 0$ & $9 / 26 / 27$ & $7 / 16 / 17$ & $2 / 10 / 10$ & $9 / 0 / 0$ & $0 / 26 / 0$ & $0 / 0 / 27$ \\
\hline & FR thickness $(\mu \mathrm{m})^{\mathrm{a}}$ & $229.1 \pm 18.4$ & $417.9 \pm 111.0$ & $402.7 \pm 109.7$ & $445.7 \pm 107.9$ & $229.8 \pm 13.5$ & $381.4 \pm 45.8$ & $515.9 \pm 59.4$ \\
\hline & IR thickness $(\mu \mathrm{m})^{a}$ & $0.0 \pm 0.0$ & $150.0 \pm 91.5$ & $138.7 \pm 91.7$ & $170.7 \pm 87.4$ & $0.0 \pm 0.0$ & $121.6 \pm 39.0$ & $227.4 \pm 59.0$ \\
\hline & OR thickness $(\mu \mathrm{m})^{a}$ & $229.1 \pm 18.4$ & $267.8 \pm 33.4$ & $264.0 \pm 34.6$ & $274.8 \pm 29.9$ & $229.8 \pm 13.5$ & $259.8 \pm 21.5$ & $288.3 \pm 33.1$ \\
\hline & ONL thickness $(\mu \mathrm{m})^{a}$ & $132.9 \pm 10.7$ & $164.7 \pm 30.9$ & $162.6 \pm 30.4$ & $168.3 \pm 31.4$ & $130.8 \pm 21.9$ & $156.2 \pm 18.4$ & $184.0 \pm 29.6$ \\
\hline & $\begin{array}{l}\text { ISOSRPE thickness } \\
(\mu \mathrm{m})^{\mathrm{a}}\end{array}$ & $96.2 \pm 7.7$ & $103.3 \pm 10.9$ & $101.4 \pm 10.5$ & $106.7 \pm 10.8$ & $99.0 \pm 12.9$ & $103.6 \pm 7.6$ & $104.4 \pm 12.5$ \\
\hline & Glaucoma (n) & 0 & 21 & 21 & 0 & 7 & 10 & 4 \\
\hline & Choroidal effusion (n) & 0 & 13 & 10 & 3 & 0 & 9 & 4 \\
\hline
\end{tabular}

a Values indicate mean \pm standard deviation, ${ }^{b}$ Values indicate the spherical equivalent BCVA: Best corrected visual acuity, Dpt: Diopter, FR: Full retina, IR: Inner retina, ISOSRPE: Distance from external limiting membrane to Bruch Membrane; LogMAR: Logarithm of the Minimum Angle of Resolution, N: Nanophthalmos, OCT: Optical coherence tomography, ONL: Outer nuclear layer, OR: Outer retina, PM: Posterior microphthalmos. 


\section{Imaging features}

The individual foveal centre could be localized in each eye due to the fact that even type 3 eyes revealed a distinct widening of the ONL and lengthening of the photoreceptor outer segments (Figure 1). Overall, nine eyes of five patients were assigned to type 1 central retinal structure, 26 eyes of 13 patients to type 2, and 27 eyes of 15 patients to type 3 (Table 1). The OCT type classification was symmetrical in all patients where both eyes were included. There was no different distribution of diagnosis (nanophthalmos vs. posterior microphthalmos, $P=0.663)$, gender $(P=0.542)$, or age $(P=676)$ between the three OCT types. However, significant different refractive error $(P<0.001)$ and axial length $(P<0.001)$ measurements were found between the different central retinal structure types (Table 1). If the axial length was below $17.8 \mathrm{~mm}$, a normal foveal configuration (i.e., OCT type 1) was absent.

Apart from the normal foveal configuration with absence of IR, type 1 eyes also had normal retinal layer thickness measurements of OR $(P=0.888)$ compared to controls (Table 1), comprising both ONL $(P=0.800)$ and ISOSRPE $(P=0.565)$. According to the OCT type classification, the central IR thickness increases from type 2 to type 3 $(P<0.001)$. There was also a trend to thickening of the central OR with increasing OCT type $(P<0.001)$. This effect was based on ONL measures $(P<0.001)$ as ISOSRPE did not significantly differ between the three OCT types $(P=0.572)$. As full retinal thickness consists of IR and OR, it was also elevated in type 2 and especially type 3 eyes compared to type 1 eyes and controls $(P<0.001$, Table 1$)$. In type 3 eyes, the full retinal thickness in the foveal centre even surmounted the perifoveal retina leading to the impression of a retinal fold (Figure 1).

Despite irregular central retinal morphology, tilting of the OCT scan led to parafoveal hypo- and hyperreflective artefacts (Figure 2 first row), which is thought to derive from intact Henle fibre layer and to represent a physiologic finding.[29] Based on other imaging modalities (AF and/or two-wavelength AF MPOD measures), all eyes also revealed macular pigment in a normal distribution (Figure 2 second row). In eyes with presence of IR in the foveal centre (i.e., OCT type 2 and 3), the foveal avascular zone was absent indicated by flow signal in both the superficial and the deep vascular network (Figure 2 third row).

\section{Disease-associated complications}

Narrow angle glaucoma and choroidal effusion was present in 21 and 13 eyes, respectively (Table 1). Eyes with narrow angle glaucoma were present in all three different OCT types, but only coincided with the diagnosis of nanophthalmos. In contrast, choroidal effusion was only present in eyes with alterations in foveal structure (i.e., type 2 and/or type 3), but did not show any differences in diagnosis-specific frequency $(P=0.468$, Table 1). Nevertheless, neither glaucoma ([mean $\pm S D]$; glaucoma, $17.04 \pm 1.69 \mathrm{~mm}$; no glaucoma nanophthalmos, $16.37 \pm 1.10 \mathrm{~mm} ; P=0.157)$ nor choroidal effusion (choroidal effusion, $16.51 \pm 1.01 \mathrm{~mm}$; no choroidal effusion, $16.52 \pm 1.35 \mathrm{~mm} ; \mathrm{P}=0.388$ ) were associated with significantly different axial measurements (Supplementary Figure S1). In our cohort, anterior chamber depth of 
glaucoma eyes $(1.78 \pm 0.44 \mathrm{~mm})$ did not differ from other nanophthalmos eyes $(1.81 \pm 0.13 \mathrm{~mm} ; \mathrm{P}=0.785)$. As remarkable result, choroidal effusion was highly correlated to more severe visual impairment $(1.08 \pm 0.72$ vs. $0.40 \pm 0.03 ; P<0.001)$.

Further investigation of features correlated to disease-associated complications can be found in Supplementary Figure S1).

\section{Inter-eye correlation}

In patients with both eyes included $(n=29)$, the diagnosis (nanophthalmos vs. posterior microphthalmos), the OCT type, and the presence of glaucoma $(n=9)$ was always similar in both eyes, while choroidal effusion presented binocularly in five and monocularly in two of these patients. In the latter, the partner eye approximately revealed similar measurements for axial length and scleral thickness. In terms of central retinal layer measures, there was a distinct inter-eye correlation (Figure 3). Similar findings could be made for axial length, refractive error, and anterior chamber (Figure 3). In contrast, there were three outliers in the inter-eye correlation of BCVA, that could be explained by the presence of choroidal effusion in both eyes and/or the eye with more severely impaired visual acuity (Figure 3 and Supplementary Table S1). More information about disease associated complications can be found in Supplementary Results.

\section{Evaluation of functionally relevant parameters}

For predictive modelling of BCVA in patients, we excluded all eyes with choroidal effusion. Hereby, multiple putative predictors were initially considered including demographic characteristics (age), macrostructural configurations (refractive error, axial length, anterior camber depth, and scleral thickness), as well as central OCT layer thickness measures (IR, ONL, and ISOSRPE). In the linear regression analysis, age, refractive error, axial length, and IR thickness showed a significant correlation with BCVA, while features like anterior chamber depth, scleral thickness, ONL and ISOSRPE thickness did not correlate significantly (Figure 4).

However, since features might exhibit a significance in a multilinear model despite not showing any real correlation in a bivariate analysis, we fitted OLS models for all possible combinations of features. The model with the lowest average MSE $(0.035 \pm 0.022)$ comprised age, refractive error, axial length, and ISOSRPE. The adjusted coefficient-of-determination (adjusted R2) was 0.617 (F-statistic, 11.46; $\mathrm{P}<0.001$ ), indicating that the chosen predictors allow explaining $61.7 \%$ of the variability in BCVA measures in eyes without choroidal effusion. In this OLS model, age, axial length and ISOSRPE thickness remained significant factors (Supplementary Table S3). Furthermore, the residuals did not show systematic tendencies indicating the reliability of the model parameters (Supplementary Figure S2).

\section{Inter-Rater Reliability}

The image grading by two independent readers (PLM and AA) revealed high inter-rater reliability. Concerning the central retinal structure types, both readers agreed to the 
classification of each eye. For retinal thickness measures, the measurements showed excellent values for ICC and low CV as well as CoR (i.e., with 0.95 probability, the absolute differences between two ratings would lie below this value, Supplementary Table S4). Also, the Bland-Altman plots did not reveal systematic inter-rater discrepancies (Supplementary Figure S3). The inter-reader variability significantly increased with larger values for measurements of IR ( $\rho=0.356, P=0.004)$ according to Spearman's rank correlation coefficient $(\rho)$ for absolute differences and mean values.

Full retinal thickness $(\rho=0.040, P=0.758)$, OR $(\rho=0.134, P=0.298)$, ONL $(\rho=0.081$, $P=0.532)$, and ISOSRPE $(\rho=0.049, P=0.707)$ did not show this correlation. 


\section{DISCUSSION}

Using modern imaging technology, this study graded and evaluated the foveal configuration of patients with nanophthalmos or posterior microphthalmos ranging from normal foveal structure to foveal hypoplasia characterized by persisting inner retinal layers, misshaped or missing foveal pit, and absent FAZ. Despite this variability, the presentation of outer retinal layers, macular pigment distribution and density, and Henle fibre layer was consistently preserved indicating the complexity of foveal development and pathophysiology of both disease entities. Further evaluation revealed a high functional relevance of demographic, clinical and imaging parameters.

Choroidal effusion had the most significant impact on visual acuity in our cohort (Supplementary Figure S1 and Figure 3). It has been suggested that the thickened sclera in patients with nanophthalmos and posterior microphthalmos causes impaired venous drainage and secondarily choroidal engorgement.[13] Although inter-eye correlation of monocular affected individuals in our cohort did not show an association of choroidal effusion and scleral thickness (likely due to the low number of $n=2$ ), it has been supported by animal models, where vortex vein occlusion led to localized congestion of a corresponding segment of iris, ciliary body, and choroid.[30] Choroidal effusions can occur and/or worsen spontaneously or with intraocular surgery in affected patients. The latter was reported to be associated with a rapid drop in intraocular pressure that leads to reduced tissue turgor that ordinarily would oppose movement of fluid from the intravascular to the extravascular space.[13] Therefore, it is worth considering special precautions during interocular interventions in patients with nanophthalmos or posterior microphthalmos.[13]

Apart from this complication, the presented findings provide evidence for the high functional relevance of axial length and refractive error (spherical equivalent), which had an even greater feature importance on the BCVA prediction model than the central retinal layer thickness measures or other global measurements such as scleral thickness. This might be traced back to the very linear correlation of axial length with BCVA (Figure 4) indicating that axial length (and indirectly refractive error) not only defines the diagnosis and is the most obvious disease characteristic,[12] but might also provide the best approximation of individual disease severity in the absence of complication. Even if it had historically been described to be associated without obvious posterior structural defects,[1] digital imaging revealed abnormal foveal structure in patients with nanophthalmos and posterior microphthalmos.[14,17-19,31-33] In our investigation, different foveal structures (i.e., OCT types 1,2 and 3) could be differentiated that were partly associated with axial length. The foveal retinal layer thickness measures were associated with a significant impact on BCVA (Figure 4). However, the feature importance did not reach the level of axial length or investigations in other retinopathies.[34,35] This might be traced back to possible differential functional importance of foveal microstructure on BCVA in nanophthalmos and posterior microphthalmos as further discussed below. The significant impact of age on BCVA (Figure 4 and Table 2) might indicate a combination of progressive disease, increasing lens opacification and/or lower number of aged patients. In total, the findings provided 
evidence for the functional relevance of demographic, clinical, and microstructural data explaining $61.7 \%$ of the variance in BCVA measures in eyes without choroidal effusion.

Inner retinal folds were occasionally found in our patients (OCT type 3). Similar to spectral domain OCT findings in posterior microphthalmos of prior studies,[17-19] only inner retinal layers were elevated over the level of parafoveal vitreoretinal surface. Other studies reported that the folds include all retinal layers in both disease entities.[14,31-33] However, these discrepancy might be attributed to the low resolution of time-domain systems, which was used in the latter. Two different hypotheses have been postulated for how the excessive foveal tissue may develop: (I) The different embryologic origins of RPE and neurosensory retina might be a reason. The thickened sclera in both diseases may limit RPE growth whereas the neurosensory retina grows normally leading to a rise of the excessive retinal tissue. $[14,17,31,32]$ Given our results that the outer retina was not excessively thickened in these cases, this hypothesis seems unlikely. (II) The increased growth of the retinal vessels that are distributed anteriorly to the neural elements of the retina cause the fold to develop. This hypothesis was stated for posterior microphthalmos based on the findings of vascular tortuosity and the presence of cysts.[18] However, we did not find these alterations in our cohort.

As expected in healthy subjects, the ONL and photoreceptor outer segments of all included patients were elongated towards the presumed fovea. Depending on the thickness of abnormally present inner retinal layers, a foveal pit was still identifiable (OCT type 2) or a central thickening could be found at this location (OCT type 3 ). In this 'pseudo-fold', the thickness of inner retinal layers however was the same as in the parafoveal area (Figure 1). This might be explained by at least two independent developmental processes that lead to the formation of the foveal structure: One process affects the spatial spreading and differentiation of the outer retinal layers; the other process leads to the development and distribution of inner retinal cells and synapses. In fact, histopathology studies revealed that as a first sign of foveal development, the central photoreceptor layer exclusively contains cones by gestation week 22 , while the foveal depression does not deepen before birth.[36,37] This indicates that the development of the specific foveal outer retina begins very early and differentiation of foveal cones (also after birth) consisting of elongation, increasing foveal density, and formation of basal axon processes (Henle fibres) might be determined a long time before birth. In contrast, the specific centrifugal displacement of the inner retina happens later from birth until 15 months.[36,37] Based on our observations, the inner retinal displacement seems to be partly altered in most patients with nanophthalmos and posterior microphthalmos (OCT type 2 and 3 ) and might be linked to the axial growth of the eye that is a characteristic feature of these disease entities.[12]

Given the foveal hypoplasia in nanophthalmos and posterior microphthalmos, Henle fibre layer might be expected to be less developed as well. However, OCT artefacts in the inner region of the outer nuclear layer (Figure 2) that are thought to derive from Henle fibre layer were present in our cohort. Also, MPOD measurements and AF revealed normal levels and distribution of macular pigment. which is postulated to be spatially connected with Henle fibre layer.[23] Thereby the development of Henle fibre 
layer and xanthophyll accumulation in the central macula might be hypothesized to be more related to the formation of the outer rather than inner retinal layers.

Up to now, an absent or rudimentary FAZ has only been reported in four cases of nanophthalmos,[15] in contrast to posterior microphthalmos where it has been more commonly described. $[3,16]$ Of note, these previous descriptions were based on fluorescein angiography findings that does not allow for differentiating of different vascular plexus within the retina. By using OCT-A, we could evaluate the flow signals in a three-dimensional resolution. We found that both superficial and deep capillary plexuses are affected in eyes of both disease entities with central presence of inner retinal layers (OCT type 2 and 3). Similar OCT-A findings were described for Alport maculopathy as causative for the associated reduced visual acuity,[35] while for Stickler syndrome no significant correlation between visual acuity and foveal thickness of abnormally present inner retinal layers in mild retinal hypoplasia and absence of FAZ could be found.[38]

Interestingly, BCVA of $\leq 0.0$ LogMAR ( $\geq 20 / 20$ Snellen equivalent) was found in one eye of OCT type 2 group and even one eye of OCT type 3 group in our cohort. Moreover, a BCVA of $\leq 0.3$ LogMAR ( $\geq 20 / 40$ Snellen equivalent) was found in eight, six and three eyes assigned to OCT type 1,2 and 3, respectively. This level of visual acuity had been stated to be unachievable for patients with nanophthalmos and posterior microphthalmos despite reports in line with our findings.[14,17] To summarize, the abnormal foveal architecture may limit visual potential, but with a high inter-individual variability. Apart from cases with complications (like choroidal effusion, Figure 2),[39] the diagnosis of nanophthalmos or posterior microphthalmos is not generally associated with low visual outcome and should therefore not discourage clinicians to e.g. perform cataract surgery if symptoms and lens opacity otherwise indicate to do so.

Limitations of the study include the number of study participants; although our cohort is the largest to date. As one of our inclusion criteria was high quality OCT images acquired as part of routine care, we may have a selection bias, where cases with better fixation, visual acuity or ocular media clarity, were included. However, the high variability of phenotypic manifestation did not influence the inter-rater agreement (Supplementary Figure S3). Due to the retrospective character, interesting aspects of retinal function like localized sensitivity derived from fundus-controlled perimetry or dark adaptation could not be investigated. Future prospective studies that systematically evaluate change of foveal structure and function over time, and additional studies in patients with a wider spectrum or more severe disease manifestation might be warranted, as imaging technology improves.

In conclusion, this study evaluated an extended spectrum of retinal alterations and identified novel imaging features in the largest cohort of patients with nanophthalmos or posterior microphthalmos to date. The study gives further insights into the physiology of foveal development, the process of emmetropisation and the complex pathogenesis of nanophthalmos and posterior microphthalmos. The results showed the importance of complications, demographic and structural parameters on visual acuity explaining the majority of the high interindividual variability of BCVA that might support clinicians in patient assessment. 


\section{ACKNOWLEDGEMENTS / DISCLOSURES}

Funding: This work was supported by the German Research Foundation (grant \# MU4279/2-1 to PLM), the United Kingdom's National Institute for Health Research of Health's Biomedical Research Centre for Ophthalmology at Moorfields Eye Hospital and UCL Institute of Ophthalmology (support to AT and CE). The views expressed are those of the authors, not necessarily those of the Department of Health. The funder had no role in study design, data collection, analysis, or interpretation, or the writing of the report.

Competing Interests: No financial disclosures exist for any author.

Contributorship Statement: PLM and CE researched literature, conceived the study, and gained ethical approval. PLM, ARW, PK, MM, LW, DS, PF, MM, CP, AT and CE were involved in patient recruitment. PLM, TT and AA were involved in data analysis. PLM and TT developed the statistical protocol. PLM wrote the first draft of the manuscript. All authors reviewed and edited the manuscript and approved the final version of the manuscript. 


\section{REFERENCES}

1 Cross HE, Yoder F. Familial nanophthalmos. Am J Ophthalmol 1976;81:300-6.

2 Othman MI, Sullivan SA, Skuta GL, et al. Autosomal dominant nanophthalmos (NNO1) with high hyperopia and angle-closure glaucoma maps to chromosome 11. Am J Hum Genet 1998;63:1411-8. doi:10.1086/302113

3 Khairallah M, Messaoud R, Zaouali S, et al. Posterior segment changes associated with posterior microphthalmos. Ophthalmology 2002;109:569-74. doi:10.1016/s0161-6420(01)00996-4

4 Sundin OH, Leppert GS, Silva ED, et al. Extreme hyperopia is the result of null mutations in MFRP, which encodes a Frizzled-related protein. Proc Natl Acad Sci 2005;102:9553-8. doi:10.1073/pnas.0501451102

5 Warburg M. Genetics of microphthalmos. Int Ophthalmol 1981;4:45-65.

6 Bergada I, Schiffrin A, Abu Srair H, et al. Kenny syndrome: description of additional abnormalities and molecular studies. Hum Genet 1988;80:39-42. doi:10.1007/BF00451452

7 Yardley J, Leroy BP, Hart-Holden N, et al. Mutations of VMD2 Splicing Regulators Cause Nanophthalmos and Autosomal Dominant Vitreoretinochoroidopathy (ADVIRC). Invest Ophthalmol Vis Sci 2004;45:3683. doi:10.1167/iovs.04-0550

8 Sugar HS, Thompson JP, Davis JD. The Oculo-Dento-Digital Dysplasia Syndrome*. Am J Ophthalmol 1966;61:1448-51. doi:10.1016/00029394(66)90484-3

9 Zenteno JC, Buentello-Volante B, Ayala-Ramirez R, et al. Homozygosity mapping identifies the Crumbs homologue 1 ( Crb1) gene as responsible for a recessive syndrome of retinitis pigmentosa and nanophthalmos. Am J Med Genet Part $A$ 2011;155:1001-6. doi:10.1002/ajmg.a.33862

10 Ayala-Ramirez R, Graue-Wiechers F, Robredo V, et al. A new autosomal recessive syndrome consisting of posterior microphthalmos, retinitis pigmentosa, foveoschisis, and optic disc drusen is caused by a MFRP gene mutation. Mol Vis 2006;12:1483-9.http://www.ncbi.nlm.nih.gov/pubmed/17167404 (accessed 6 Dec 2019).

11 O'Grady RB. Nanophthalmos. Am J Ophthalmol 1971;71:1251-3.

12 Singh OS. Nanophthalmos: guidelines for diagnosis and therapy. In: Albert DM, Jakobiec FA, eds. Principles and Practice of Ophthalmology. Philadelphia: : WB Saunders 2000. 2846-2859.

13 Ryan EA, Zwaan J, Chylack LT. Nanophthalmos with uveal effusion: clinical and embryologic considerations. Ophthalmology 1982;89:1013-7. doi:10.1016/s01616420(82)34686-2 
14 Bijlsma WR, van Schooneveld MJ, Van der Lelij A. Optical coherence tomography findings for nanophthalmic eyes. Retina 2008;28:1002-7.

doi:10.1097/IAE.0b013e31816d400c

15 Walsh MK, Goldberg MF. Abnormal foveal avascular zone in nanophthalmos. Am J Ophthalmol 2007;143:1067-8. doi:10.1016/j.ajo.2007.01.051

16 Spitznas M, Gerke E, Bateman JB. Hereditary Posterior Microphthalmos With Papillomacular Fold and High Hyperopia. Arch Ophthalmol 1983;101:413-7. doi:10.1001/archopht.1983.01040010413014

17 Almoallem B, Arno G, De Zaeytijd J, et al. The majority of autosomal recessive nanophthalmos and posterior microphthalmia can be attributed to biallelic sequence and structural variants in MFRP and PRSS56. Sci Rep 2020;10:1-10. doi:10.1038/s41598-019-57338-2

18 Jackson TE, Yang YC, Shun-Shin GA. Spectral domain optical coherence tomography findings in retinal folds associated with posterior microphthalmos. $J$ Am Assoc Pediatr Ophthalmol Strabismus 2012;16:389-91. doi:10.1016/j.jaapos.2012.02.020

19 Nowilaty SR, Mousa A, Ghazi NG. The posterior pole and papillomacular fold in posterior microphthalmos: novel spectral-domain optical coherence tomography findings. Ophthalmology 2013;120:1656-64. doi:10.1016/j.ophtha.2013.01.026

20 Mukhopadhyay R, Sergouniotis PI, Mackay DS, et al. A detailed phenotypic assessment of individuals affected by MFRP-related oculopathy. Mol Vis 2010;16:540-8. doi:10.2337/diacare.14.1.75

21 Turnbull C, Scott RH, Thomas E, et al. The 100000 Genomes Project: bringing whole genome sequencing to the NHS. BMJ 2018;361:k1687. doi:10.1136/bmj.k1687

22 Delori FC. Autofluorescence method to measure macular pigment optical densities fluorometry and autofluorescence imaging. Arch Biochem Biophys 2004;430:156-62. doi:10.1016/j.abb.2004.05.016

23 Müller PL, Müller S, Gliem M, et al. Perception of Haidinger Brushes in Macular Disease Depends on Macular Pigment Density and Visual Acuity. Invest Ophthalmol Vis Sci 2016;57:1448-56. doi:10.1167/iovs.15-19004

24 Trieschmann M, Heimes B, Hense HW, et al. Macular pigment optical density measurement in autofluorescence imaging: comparison of one- and twowavelength methods. Graefes Arch Clin Exp Ophthalmol 2006;244:1565-74. doi:10.1007/s00417-006-0289-3

25 Creuzot-Garcher C, Koehrer P, Picot C, et al. Comparison of two methods to measure macular pigment optical density in healthy subjects. Invest Ophthalmol Vis Sci 2014;55:2941-6. doi:10.1167/iovs.13-13568 
26 Team RC, R CT. R: A language and environment for statistical computing. R Foundation for Statistical Computing. 2015.https://www.r-project.org/

27 Shrout PE, Fleiss JL. Intraclass correlations: Uses in assessing rater reliability. Psychol Bull 1979;86:420-8. doi:10.1037/0033-2909.86.2.420

28 Bland JM, Altman D. Statistical methods for assessing agreement between two methods of clinical measurement. Lancet 1986;327:307-10.

29 Lujan BJ, Roorda A, Knighton RW, et al. Revealing Henle's fiber layer using spectral domain optical coherence tomography. Invest Ophthalmol Vis Sci 2011;52:1486-92. doi:10.1167/iovs.10-5946

30 Hayreh SS. Segmental nature of the choroidal vasculature. Br J Ophthalmol 1975;59:631-48. doi:10.1136/bjo.59.11.631

31 Kim JW, Boes DA, Kinyoun JL. Optical coherence tomography of bilateral posterior microphthalmos with papillomacular fold and novel features of retinoschisis and dialysis. Am J Ophthalmol 2004;138:480-1. doi:10.1016/j.ajo.2004.03.015

32 ARAS C, OZDAMAR A, USTUNDAG C, et al. OPTICAL COHERENCE TOMOGRAPHIC FEATURES OF PAPILLOMACULAR FOLD IN POSTERIOR MICROPHTHALMOS. Retina 2005;25:665-7. doi:10.1097/00006982-20050700000021

33 Erdöl H, Kola M, Türk A, et al. Ultrasound Biomicroscopy and OCT Findings in Posterior Microphthalmos. Eur J Ophthalmol 2008;18:479-82. doi:10.1177/112067210801800331

34 Russell RA, Crabb DP, Malik R, et al. The relationship between variability and sensitivity in large-scale longitudinal visual field data. Invest Ophthalmol Vis Sci 2012;53:5985-90. doi:10.1167/iovs.12-10428

35 Zhioua Braham I, Smaoui S, Boukari M, et al. Swept-source OCT and OCT angiography findings in Alport maculopathy. Acta Ophthalmol 2018;96:142-142. doi:10.1111/aos.13972_530

36 Hendrickson AE, Yuodelis C. The morphological development of the human fovea. Ophthalmology 1984;91:603-12. doi:10.1016/s0161-6420(84)34247-6

37 Yuodelis C, Hendrickson A. A qualitative and quantitative analysis of the human fovea during development. Vision Res 1986;26:847-55. doi:10.1016/00426989(86)90143-4

38 Matsushita I, Nagata T, Hayashi T, et al. Foveal Hypoplasia in Patients with Stickler Syndrome. Ophthalmology 2017;124:896-902. doi:10.1016/j.ophtha.2017.01.046

39 Auffarth GU, Blum M, Faller U, et al. Relative anterior microphthalmos: 
Morphometric analysis and its implications for cataract surgery. Ophthalmology 2000;107:1555-60. doi:10.1016/S0161-6420(00)00240-2 


\section{FIGURE CAPTIONS}

\section{Figure 1: Image grading of central retinal structure}

The exemplary optical coherence tomography (OCT) images in the top row demonstrate the definition of the three different types of foveal depression in patients with nanophthalmos and posterior microphthalmos: Type 1 represents normal foveal structure featuring extrusion of the inner retinal layers, foveal pit, widening of the outer nuclear layer, and lengthening of the photoreceptor outer segments. Cases with incomplete extrusion of the inner retinal layers and a definite foveal pit were assigned to type 2. Type 3 indicates absence of a foveal pit. Below, the central area of the type 2 OCT scan is magnified to highlight the segmentation of the retinal layers. Layer thickness was measured in the foveal centre (green dotted line) from internal limiting membrane to Bruch's membrane for 'full retina (FR)', from internal limiting membrane to inner border of outer nuclear layer for 'inner retina (IR)' (dark green), from inner border of outer nuclear layer to Bruch's membrane for 'outer retina (OR)', from inner border of outer nuclear layer to external limiting membrane for 'outer nuclear layer (ONL)' (yellow), and from the external limiting membrane to Bruch's membrane comprising 'photoreceptor inner and outer segments as well as RPE cells (ISOSRPE)' (purple).

\section{Figure 2: Imaging features}

A representative tilted central optical coherence tomography (OCT) scan (A), infraredreflectance image (B), fundus autofluorescence (AF) image (C), macular pigment optic density (MPOD) image (D), and results of OCT-Angiography (OCT-A, E-G) centred on the fovea are shown. In the tilted OCT scan, the physiologic parafoveal hypo- (white dots) and hyperreflective (black dots) artefacts in the inner region of the outer nuclear layer become visible. Corresponding to the foveal MPOD peak (central bright area in the MPOD image), the AF image shows central shadowing. In the central OCT-A BScan (E), blood flow is color-coded in red that revealed presence of vasculature in the inner retinal layers at the presumed foveal location. The absence of a foveal avascular zone was correspondingly present in the superficial $(F)$ and the deep vascular network $(\mathrm{G})$.

\section{Figure 3: Inter-eye correlation}

There was a distinct correlation between the right (OD, $x$-axis) and left (OS, $y$-axis) eye for optical coherence tomography layer thickness measures (A-D) as well as global measurements like axial length $(E)$, refractive error (spherical equivalent) $(F)$, and anterior (ant.) chamber depth (G). Here, the absence (grey dot) or presence of choroidal effusion in one (pink square, always right eye) or both (red cross) eyes neither had impact on the inter-eye correlation nor were associated with a specific range of measurements. For best corrected visual acuity (BCVA, H), three outliers were present that were associated with the highest visual impairment and the presence of choroidal effusion. FR: Full retina, IR: Inner retina, ISOSRPE: Distance from external limiting 
membrane to Bruch Membrane, ONL: Outer nuclear layer, OR: Outer retina, LogMAR: Logarithm of the Minimum Angle of Resolution.

\section{Figure 4: Linear regression analysis of individual features}

The demographic, clinical and imaging (including optical coherence tomography, OCT) features of patients not suffering from choroidal effusion were correlated with their respective best corrected visual acuity (BCVA) values (in Logarithm of the Minimum Angle of Resolution, LogMAR). For each linear fit (red line), the $95 \% \mathrm{Cl}$ (red area), as well as the Pearson Correlation Coefficient (PCC) and $P$ value of the fit are shown. IR: Inner retina, ISOSRPE: Distance from external limiting membrane to Bruch Membrane, ONL: Outer nuclear layer. 